\title{
Management of Specific Complications after Congenital Heart Surgery (I)
}

\author{
A. Sánchez Andrés' ${ }^{1}$ C. González Miño², E. Valdés Diéguez ${ }^{3}$, L. Boni ${ }^{3}$, J. I. Carrasco Moreno \\ ${ }^{1}$ Pediatric Cardiology Unit, Hospital Universitario y Politécnico La Fe, Valencia, Spain \\ ${ }^{2}$ Pediatric Intensive Care Unit, Hospital General de Castellón, Castellon, Spain \\ ${ }^{3}$ Pediatric Surgery Unit, Hospital Universitario y Politécnico La Fe, Valencia, Spain \\ Email: tonisanchan@hotmail.com
}

Received 18 February 2015; accepted 12 March 2015; published 17 March 2015

Copyright (C) 2015 by authors and Scientific Research Publishing Inc.

This work is licensed under the Creative Commons Attribution International License (CC BY). http://creativecommons.org/licenses/by/4.0/

(c) (i) Open Access

\begin{abstract}
In addition to the general consequences of surgery and cardiopulmonary by-pass, lesion-specific complications can occur after surgery for congenital heart disease. It is important for the pediatric intensive care specialist to fully understand the preoperative anatomy and the intraoperative details of these patients. This allows a timely and appropriate treatment of general and lesion-specific complications. In this article we provide a list of commonly-performed surgical procedures and possible associated problems to be anticipated in the early postoperative period. Then it follows a discussion about the diagnosis and management of these complications, based on their pathophysiological features.
\end{abstract}

\section{Keywords}

Congenital Heart Diseases, Pediatric Heart Surgery, Postoperative

\section{Introduction}

1) Congenital heart disease is the most common birth defect. Recent prevalence estimates range from 6 to 10 per 1000 live births. Many of these infants require surgery to correct or palliate their heart defect; several of them require surgery in the newborn period [1].

2) Optimal management of the postoperative pediatric cardiac surgical patient requires a thorough understanding of patient anatomy, physiology, surgical procedure and clinical condition [2].

3) Cardiopulmonary bypass, specific surgical procedure, anesthesia and other medications have the potential to produce multisystemic effects [2]. Postoperative care focuses on anticipating potentially deleterious effects and a timely intervention to restore cardiopulmonary homeostasis and prevent end organ damage [3].

4) Multidisciplinary approach: as these patients are currently managed in intensive care units, a multiduscip- 
linar collaborative effort is required to achieve a better outcome. An accurate and timely determination of cardiac output, systemic $\mathrm{O}_{2}$ delivery, and tissue oxygenation is essential to optimizing outcomes [4].

5) Severity of illness scores have been developed to predict postoperatory outcomes, based on bed side collected data, such us lactate levels or drugs employed for haemodynamic support (VIS score); recently, Kyle G. Miletic et al. [3] have reported a novel vasoactive-ventilation-renal score (VVR) incorporating postoperative markers of cardiovascular, pulmonary and renal dysfunction, the three most commonly affected organ systems in children after surgery for congenital heart disease (CHD).

6) Relative to the amount of information avaliable about CPB and general complications after pediatric cardiac surgery, updated literature about lesión specific complications is lagging [5].

The main objective of this article is to provide a list of commonly-performed surgical procedures and possible associated problems to be anticipated in the early postoperative period. The diagnosis and management of these complications, based on their pathophysiological features are also discussed.

\section{Complications after Cardiac Procedures}

The following tables show the most common problems to be anticipated in the early postoperative period according to the general pathophysiological mechanism underlying the congenital heart disease, as well as the specific procedure performed.

1) Repair of left to right shunts (Table 1).

2) Repair of right-sided obstructions (Table 2).

3) Repair of left-sided obstructions (Table 3).

4) Palliative procedures (Table 4).

5) Miscellaneous: including procedures for anatomic and physiologic correction of D-transposition of the great arteries and repair of anomalous origin of coronary artery from pulmonary artery and surgery on the mitral valve (Table 5).

\section{Diagnosis and Management of Specific Complications}

\subsection{Residual Left to Right Shunts (Table 1)}

It is the case of an incorrect septal defect closure or a residual shunt at other levels (e.g.: systemic-pulmonary collateral arteries or apical muscular VSDs), either preoperatively unrecognized or recognized but intentionally left untreated. The pathophysiology of these shunts include the development of pulmonary edema secondary to increased pulmonary blood flow, pulmonary hypertension, ventricular volume overload, and in some circumstances systemic cardiac output limitation. The most common signs and symptoms include a pulmonary (or VSD type) ejection murmur, high left atrial filling pressures, cardiomegaly with signs of increased pulmonary blood flow on chest radiograph, and variable degrees of low cardiac output [6].

Residual shunts at atrial level are rarely a problem, unless other factors increase the shunt volume. The development of congestive heart failure should guide the clinician to rule out mitral stenosis or regurgitation, small size and/or dysfunction of the systemic ventricle, and systemic ventricular outflow tract obstruction [7].

Residual shunts at ventricular or arterial level are clinically more relevant because of the systemic ventricle volume overload and the pressure and volume overload of the pulmonary circulation. In general, shunts with a Qp:Qs smaller than 1.5 - 2.1 without pulmonary hypertension are well tolerated. Larger shunts are almost always associated with congestive heart failure. Patients with large preoperative left to right shunt usually tolerate residual shunts better than patients who were cyanotic before surgery. For example, a patient with a residual ventricular septal defect after repair of tetralogy of Fallot, moves from a situation in which the left ventricle is normally loaded preoperatively to a new situation in which it is overloaded with volume. At pulmonary level, from a situation of reduced pulmonary flow we pass to the opposite scenario of pulmonary overload. These factors make the residual defect poorly tolerated [8].

Once the diagnosis of residual shunt is made, the right treatment must be chosen, balancing the risks and benefits of a surgical or a percutaneous intervention. Some injuries are not repairable and these patients need to be managed with conservative measures. Maneuvers that reduce pulmonary vascular resistances must be avoided because they increase the shunt magnitude. Examples of these are the use of high oxygen concentrations or 
Table 1. Repair of left to right shunts.

\section{$\underline{\text { General Complications }}$}

-Residual defects: leading to various degrees of left to right shunt.

-Pulmonary hypertension: this is the most predictable complication in older patients who had time to develop pulmonary vascular disease, in neonates and in patients with pulmonary venous obstruction.

-Arrhythmias: any arrhythmia can occur after any cardiac surgical procedure, but the patients with ventricular or atrioventricular septal defects have a greater risk of complete heart block (CHB) and junctional ectopic tachycardia (JET).

\section{Specific Complications}

- Repair of atrial septal defects

-Sinus-Node dysfunction.

-Acute left-ventricular failure (in older children and adults).

- Repair of ventricular septal defects

-Pulmonary Hypertension.

-Arrhythmias: JET and CHB.

-Insufficiency or stenosis of the right atrioventricular valve.

- Ligation of patent ductus arteriosus

-Recurrent laryngeal nerve injury.

-Damage or ligation of the left pulmonary artery or aorta.

- Repair of truncus arteriosus

-Pulmonary hypertension.

-Truncal valve stenosis or regurgitation.

-Right ventricular dysfunction.

- Repair of aorto-pulmonary window

-Pulmonary hypertension.

- Repair of anomalous pulmonary venous return

-Pulmonary hypertension.

-Arrhythmias.

-Residual stenosis of the pulmonary veins or anastomosis.

-High left ventricular filling pressures due to the small size of the left chambers.

hyperventilation. Inotropic support, mechanical ventilation, afterload reducers, and the use of diuretics are usually needed. As soon as the heart recovers from the deleterious effects of surgery, the residual shunt will become better tolerated, allowing a decrease of the medical treatment [9].

\subsection{Residual Obstruction of the Ventricular Outflow Tracts}

Residual obstructions should be sought after any surgery on the ventricular outflow tracts (Tables 2-3), such as repair of subaortic stenosis, aortic or pulmonary valve commisurotomy, Fallot repair. Furthermore, systemic ventricle obstruction may occur after repair of atrio-ventricular septal defects or after interventions that reduce the ventricular volume preload when the underlying anatomy includes subvalvular hypertrophy (often septal). Ventricular hypertrophy can cause obstruction of the foramen narrowing subvalvular ventricular bulb.

Signs and symptoms of systemic ventricle obstruction include an ejection systolic murmur, left atrial hypertension, 
Table 2. Repair of right-sided obstructions.

\section{General Complications}

- Residual stenosis or regurgitation.

- Right ventricular dysfunction: both systolic and diastolic. The latter is more common due to the right ventricle hypertrophy and its poor compliance, particularly if a right ventriculotomy is performed.

\section{$\underline{\text { Specific Complications }}$}

- Repair of Fallot's tetralogy and its variants

-Right ventricular dysfunction.

-Arrhythmias: JET, CHB.

-Pulmonary regurgitation.

-Residual pulmonary stenosis.

-Residual ventricular septal defect.

- Repair of absent pulmonary valve syndrome

-Tracheobronchomalacia.

- Repair of pulmonary atresia with intact ventricular septum

-Right ventricular dysfunction.

-Myocardial ischemia (in case of right ventricular dependent coronary circulation).

-Circular shunt: if it is used both systemic to pulmonary fistula type modified

Blalock-Taussig shunt and a patch on the right ventricular outflow tract.

-Hypoxia (particularly in the first postoperative hours)

pulmonary edema and a low cardiac output state (according to the degree of the residual defect) [10].

In the interpretation of the diagnostic tests (including echocardiography and cardiac catheterization), special emphasis must be placed in the anatomical details of the obstruction, especially in presence of ventricular dysfunction, when a severe stenosis may not produce an accordingly severe gradient. Unlike adult patients with systemic ventricle obstruction, the use of beta-adrenergic agonists is not usually associated with myocardial ischemia, provided that cardiac output and myocardial oxygen delivery are assured.

Special attention must be paid when the obstruction is dynamic, since an increase in myocardial contractility may worsen the lesion. Therefore in this setting inotropes are contraindicated. The presence of systemic ventricular obstruction is also a relative contraindication to the use of vasodilation agents. In fact systemic vasodilation in presence of ventricular outflow obstruction may cause severe hypotension, due to the inability of the heart to increase cardiac output [11] [12].

Patients who had a significant relief of systemic outflow tract obstruction easily develop systemic arterial hypertension, due to a maintained hyperdynamic state of the heart and vessels. Treatment of hypertension is particularly important in the first 24 hours after surgery, to protect the surgical repair (sutures) and to minimize the risk of bleeding. The most effective drugs include vasodilators, beta-blockers and inhibitors of angiotensin converting enzyme (ACEi).

\subsection{Dysfunction of the Mitral or Tricuspid Valve}

Residual atrio-ventricular (AV) valve regurgitation or stenosis may occur after any reparative surgery of the valve itself or when closing a septal defect unmasks valvular stenosis in one side of the heart.

$\mathrm{AV}$ valve insufficiency results in elevated atrial pressures on the affected side. If atrial pressure is being monitored directly, it can get drawn with very prominent $\mathrm{V}$ waves and a regurgitant murmur may be listened. Because of the associated ventricular volume overload, signs of ventricular failure may develop.

$\mathrm{AV}$ valve stenosis is also associated with high pressure in the atria, but not with ventricular volume overload. Systemic or pulmonary edema is very common in $\mathrm{AV}$ valve stenosis as a consequence of systemic or pulmonary 
Table 3. Repair of left-sided obstructions.

\section{General Complications}

- Residual stenosis.

- Systemic arterial hypertension.

- Left ventricular diastolic dysfunction.

\section{$\underline{\text { Specific Complications }}$}

- Repair of aortic stenosis

-Valvotomy: residual stenosis/aortic regurgitation.

-Ross operation: myocardial ischemia.

-Konno operation: myocardial Ischemia, right ventricular outflow tract obstruction and arrhythmias.

- Repair of subaortic stenosis

-Residual stenosis.

-Iatrogenic mitral valve regurgitation.

-Arrhythmias.

- Repair of supravalvar aortic stenosis

-Residual aortic stenosis.

-Aortic regurgitation due to valve injury.

-Myocardial ischemia.

- Repair of aortic coarctation

-Residual stenosis.

-Paraplegia.

-Postcoarctectomy syndrome.

-Aortic stenosis unmasked.

-Recurrent laryngeal nerve injury.

- Repair of interrupted aortic arch

-Residual stenosis (normally at anastomosis site).

-Left bronchial compression (by the advanced aorta).

-Hypocalcemia (if DiGeorge Syndrome associated).

- Repair of mitral stenosis

-Pulmonary hypertension.

-Residual stenosis or regurgitation.

-Left ventricular dysfunction.

venous hypertension respectively. Cardiac output may be limited in case of severe A-V valve stenosis or insufficiency, especially in acute onset [6].

In case of a hemodinamically significative lesion —and if the valve is amenable of surgical repair-the patient should be brought back to the OR without delay. If the alternative is a prosthetic valve implantation, palliative medical treatment should be considered, given the poor outcome of prosthetic valves in young patients. The medical management of AV valve regurgitation focuses on afterload reduction with inotropic support when necessary. Systemic vasodilators are useful in mitral regurgitation, while diuretics may be useful in both AV valve 
Table 4. Palliative procedures.

Systemic to pulmonary shunts (Modified Blalock-Taussia, Central shunts)

- Excessive pulmonary blood flow.

- Arterial hypotension.

- Shunt thrombosis (acute hypoxemia).

Pulmonary Artery Banding

- Cyanosis.

- Excessive pulmonary blood flow.

Table 5. Miscellaneous.

Arterial switch operation for D-transposition of qreat arteries

- Myocardial ischemia and left ventricular dysfunction.

- Aortic regurgitation.

- Supravalvar pulmonary stenosis.

\section{Atrial switch operation (Mustard or Senning)}

- Tunnel stenosis (pulmonary and/or systemic venous obstruction).

- Arrhythmias.

$\underline{\text { Repair of anomalous left or right coronary artery from pulmonary artery }}$

- Myocardial failure with or w/o mitral regurgitation (secondary to ischemia).

Mitral valve surgery

- Residual stenosis or regurgitation.

- Pulmonary hypertension.

regurgitation and stenosis. Even though medical treatment of an AV valve stenosis is usually poorly effective, associated conditions (such as pulmonary hypertension) require aggressive treatment [13] [14].

\subsection{Left or Right Ventricular Diastolic Dysfunction}

Ventricular diastolic dysfunction should be expected after any cardiac surgery on a hypertrophied heart, such as after repair of obstructive lesions of either ventricular outflow tract. When a right ventriculotomy is needed (especially in a hypertrophied ventricle) both diastolic and systolic dysfunction may develop. Furthermore, due to its anatomical location, the right ventricle is particularly exposed to the operating room temperature, so that myocardial preservation by means of systemic cooling may be less effective.

Diastolic dysfunction is characterized by high pressures at atrial level and has similar characteristics to those seen in AV valve dysfunction. The presence of a residual shunt at atrial level exacerbates the symptoms of cardiac failure in case of left (systemic) ventricular dysfunction, while it may be useful in maintaining the cardiac output in case of right (pulmonary) ventricular dysfunction (e.g., TOF repair). In this case, a certain degree of cyanosis must be expected, but it is normally well tolerated.

The need to maintain high filling pressures of a ventricular chamber (see treatment here below) results in increased upstream hydrostatic pressures which, in turn, favors the extravasation of fluids and leads to pulmonary or generalized (systemic) edema, depending on the side affected [15] [16].

The first-line treatment of diastolic dysfunction is the administration of IV fluids to maintain an adequate ventricular preload, although this will come at the expense of an increase in systemic and pulmonary venous pressures. Thus, fluids administration may be limited by the consequences of pulmonary or systemic venous hypertension, such as hypoxia or severe peripheral edema and effusions respectively [17]. The use of inodilators, 
such as milrinone, is also very useful. Especially the right ventricle responds very well to the initial administration of fluids, but-as seen — high hydrostatic pressures easily lead to the appearance of pleural effusions and/or ascites. Without adequate drainage of these effusions the intra-thoracic and intra-abdominal pressures will increase. This will lead, in the first case, to higher airway pressures which will be transmitted to the pulmonary vasculature. This situation of increased pulmonary vascular resistance will negatively affect the already depressed right ventricle, thus maintaining a vicious circle, apart from altering the gas exchange. Increased intra-abdominal pressure may result in decreased renal perfusion and eventually kidney failure, complicating the fluids and electrolytes management. All this triggers a downward spiral in which, finally, cardiac output is affected and it is difficult to restore. Effective treatment includes timely drainage of pleural effusions and ascites, together with an appropriate hidroelectrolyte management. In cases of refractory right ventricular dysfunction, mechanical assistance with ECMO may be needed. In general, right ventricular diastolic function improves within days, as far as the right ventricle recovers from surgery and becomes more distensible (and assuming that the intervention restored normal or near-normal RV systolic pressures). Diastolic dysfunction can be managed in a similar way, but more likely to be associated with prolonged cardiac arrest and/or cardiac transplantation.

\subsection{Pulmonary Hypertension}

Early repair of cardiac defects characterized by increased pulmonary blood flow has reduced the incidence of postoperative pulmonary hypertension. However the use of cardiopulmonary bypass during most pediatric cardiac interventions can produce pulmonary hypertension in patients with underlying risk factors. Neonates, patients with obstruction of pulmonary veins or mitral valve dysfunction, and those with high preoperative pulmonary vascular resistance, are at higher risk. Pain, agitation, tracheal aspirations or hypoventilation may trigger a pulmonary hypertensive crisis. Signs and symptoms of pulmonary hypertension depend on the acuity of changes in lung (pulmonary vascular resistance) resistance and the underlying anatomy. Chronic pulmonary hypertension that doesn't worsen acutely with cardiopulmonary bypass may be well tolerated during the postoperative period while the acute crisis can produce severe life-threatening symptoms.

A patent foramen ovale, ASD, VSD or systemic-pulmonary shunts are responsible of the major clinical manifestation of an acute elevation in pulmonary pressure, which is the cyanosis (due to a right to left shunt). Pulmonary hypertension in absence of septal defects can cause acute right ventricular failure and low cardiac output, without significant changes in saturation. A sudden drop in blood pressure or saturation in a patient with known pulmonary vascular disease or significant risk factors for pulmonary hypertension should immediately make the clinician consider this diagnosis and consequently establish the adequate treatment. The presence of a pulmonary artery pressure catheter can help in the establishment of a diagnosis. Most commonly there will be a drop in systemic arterial pressure while pulmonary pressure will not change. The increase of pulmonary to systemic pressure ratio is diagnostic of increased pulmonary resistance.

The increase in pulmonary arterial pressure is transmitted to the right ventricle and as a consequence the interventricular septum will be shifted leftward, compromising the function of the left ventricle. Tachycardia is a common sign that shows how the heart struggles to maintain systemic output at low-volume filling. Other clinical manifestations include the sudden drop in lung compliance or the development of bronchospasm.

The most effective measure to manage patients at high risk of pulmonary hypertension in the postoperative period is prevention. The establishment and maintenance of proper analgesia and sedation, especially during painful stimuli (such as OT tube suctioning), is very important. The induction of respiratory alkalosis may be helpful in a pulmonary hypertensive crisis, but maintaining a $\mathrm{pH}$ above 7.5 for long periods of time can have deleterious effects on cerebral perfusion. Therefore, a practical approach would be to prevent and timely treat common problems that lead to hypoxia and respiratory acidosis, such as pneumothorax, selective right bronchus intubation or the presence of bronchial secretions, and try to maintain $\mathrm{pH}$ between 7.4 and 7.5. Then one would try to normalize the $\mathrm{pH}$ and reduce the sedation day by day with caution, paying attention to the appearance of any symptoms of pulmonary artery hypertension. When pulmonary hypertension persists for more than $4-7$ days, it is suggestive of the existence of residual lesions or chronic injury of the pulmonary vasculature.

If prophylactic measures fail, aggressive treatments such as nitric oxide, sildenafil, bosentan, or even mechanical circulatory support are indicated. Numerous studies have shown that low doses of nitric oxide (2 - 20 parts per million-ppm) are as effective as high doses (40 - 80 ppm) and are associated with fewer side effects, such as methemoglobinemia or secondary hypotension. Sildenafil and bosentan can be used as a transition from inhaled nitric oxide to chronic oral therapy, given that the use of exogenous NO inhibits the production of en- 
dogenous NO. Other intravenous vasodilators, including calcium channel blockers, nitrates and prostaglandins, have been used, but they have the limitations of systemic hypotension due to the lack of pulmonary selectivity. Prostacyclin has proved to be an effective drug that can reverse the changes in the pulmonary vasculature before they become permanent [18].

All these strategies are based on an attempt to dilate the pulmonary vascular bed to increase pulmonary blood flow. If this is not possible, the next option is to try to alter the balance between pulmonary and systemic vascular resistances, by increasing systemic ones:

- First of all an adequate preload should be guaranteed, for which colloid or crystalloid administration at 30 - 40 $\mathrm{mL} / \mathrm{kg}$ is useful.

- Arterial pH should be maintained around 7.45 to 7.50, and blood $\mathrm{PaCO}_{2}$ between 30 and $35 \mathrm{mmHg}$. Serum bicarbonate should be kept at the higher limit of its range. High frequency ventilation may be used to achieve these goals and sodium bicarbonate boluses or infusion may be administered if necessary.

- Simultaneously we can introduce inhaled nitric oxide (iNO) at $10-20$ ppm.

- If oxygen saturations are still low, the next step is to increase mean systemic arterial pressure to 60 - 70 mmHg (depending on the patient's age), which can be achieved with an infusion of norepinephrine (being this drug much more selective on the peripheral vascular bed than on the pulmonary one) or high-dose dopamine.

- We can try the administration of intravenous magnesium (due to its smooth muscle relaxant effect, being a calcium antagonist, and also being effective as skeletal muscle relaxant, sedative and antiarrhythmic drug) in bolus or continuous infusion to maintain serum magnesium concentrations between 3 to $5.5 \mathrm{mmol} / \mathrm{L}$.

- Finally we can use intravenous adenosine starting with a bolus of 50 microgrs $/ \mathrm{kg}$ and then infused at 25 - 50 microgrs $/ \mathrm{kg} /$ minute.

With this therapeutic strategy we can successfully manage patients with such low oxygenation rates that would usually be treated with an ECMO device, without significant differences in mortality [19].

\subsection{Acute Low Cardiac Output}

Many congenital heart defects produce pressure and volume ventricular overload, which if maintained for long time lead to myocyte damage and death. This will lead to ventricular dysfunction and eventually low cardiac output syndrome. To avoid these deleterious effects, early primary repair of cardiac defects is nowadays the preferred strategy in many centers.

Another cause of cellular damage is the relative myocardial ischemia during the aortic cross clamp phase of most cardiac operations. Coronary artery blood flow is interrupted and a cardioplegic solution (rich in potassium) is infused into them to stop the heart in diastole and to protect the myocytes. Many advances are needed to create the ideal cardioplegic solution to keep the heart viable in absence of coronary perfusion, and to prevent ventricular failure. Another factor during surgery that predispose to ventricular failure is the need to perform a ventriculotomy. This creates a scar without contractile capacity, which may lead to acute (or chronic) postoperative ventricular failure [20]. Other factors accounting for postoperative low cardiac output are: 1) residual cardiac lesion; 2) inflammatory response triggered by CPB; 3) changes in systemic vascular resistances and pulmonary vascular resistances; 4) arrhythmias and 5) cardiac tamponade [2].

The aims of the management of the patient in low cardiac output state are to reverse the cause of ventricular dysfunction, to optimize its preload, to increase the contractility with medications that do not increase oxygen consumption, and to decrease the ventricular afterload. Actually the most important aspect is to reduce the afterload, as this improves contractility and reduces myocardial oxygen consumption, although in patients with left ventricular outflow tract obstruction this measure should be applied with caution [21].

When high inotropic doses are needed and especially if the patient does not improve (with progressive increase in serum lactate and persistence of low mixed venous saturation) a circulatory mechanical support device should be implanted, to prevent the deleterious consequences of maintained low cardiac output state and multiple organ dysfunction.

In the pharmacological treatment, we will make a special mention of Levosimendan, a drug with promising results in postoperative adult patients and whose effects are currently being studied in the pediatric population, with promising results in several published clinical trials. This drug sensitizes the myocardium to the action of calcium, stabilizing the troponin molecule in the cardiac muscle and prolonging the effect of calcium on the contractile proteins. It has vasodilatory properties and has also been shown to increase cardiac output and to decrease wedge pressure and pulmonary resistance in patients with heart failure [22] [23]. 
A published study in pediatric patients [24] has shown the effectiveness of Levosimendan administration in 26 patients (21 postoperative and 5 with dilated cardiomyopathy and severe ventricular dysfunction). The doses used in continuous intravenous infusion were $0.1 \mathrm{mcgrs} / \mathrm{kg} / \mathrm{min}$ which increased after two hours to $0.2 \mathrm{mcgrs} /$ $\mathrm{kg} / \mathrm{min}$, and this was maintained for 24 hours. All patients were monitored and the administration of intravenous vasodilators (nitroprusside) was suspended during the infusion of Levosimendan. A side effect considered mild (which necessitated dose reduction) was hypotension requiring increase in ordinary inotropic infusion and increase in volume needs. Serious side effects (requiring drug suspension) were the onset of dangerous ventricular arrhythmias.

The drug's safety was demonstrated, since only one case of treatment discontinuation due to serious ventricular arrhythmia was reported. This patient was treated with additional antiarrhythmic therapy and the problem was resolved in three days. Mild adverse effects can be minimized by adjusting the arterial pressure, the central venous pressure and the serum ions prior to the drug administration and monitoring vital signs during the treatment.

The most immediate beneficial effect was the reduction of serum lactates and central venous pressure. The hemodynamic improvement in most patients allowed removing hemofiltration in those patients who were being treated with it, as well as weaning patients who were on mechanical ventricular assistance. It also showed improvement of patients as a bridge to heart transplantation. Levosimendan doses may be repeated weekly (according to its metabolite half-life).

Thus, the authors of this study conclude that patients who would mostly take advantage of Levosimendan are those with moderate-severe heart failure on waiting list for cardiac transplantation, because the drug would allow them to maintain their clinical situation and avoid worsening. On the other hand, patients with postoperative acute-moderate heart failure also benefit from the use of Levosimendan, in order to achieve myocardial recovery as fast as possible with the lowest dose of intravenous inotropes.

\section{Discussion}

Compared to adults, children need a much closer monitorization in the postoperative period, due to the onset of minute by minute changes related to conditions such as age, maturation, growth, development, preoperative condition and type of surgery performed. This observation currently requires a highly specialized staff who is familiar with the management of these patients. Postoperative course is different weather the patient is a newborn, a child or an infant. Especially the newborn due to conditions of immaturity, mainly cardiovascular, respiratory, neurological and renal levels can develop a greater number of complications increasing morbidity and mortality.

Preoperative patient condition is very important since children with complex heart anomalies and varying degrees of heart failure, shock or respiratory failure, are patients at risk of a storm and difficult to handle postoperative. Not surprisingly, these patients need more complex surgeries and therefore their mortality rate is higher. Currently, most of the centers worldwide report an mortality about $6 \%$, while the overall mortality for adults is around $3 \%$.

Pediatric cardiac surgery has improved considerably during the last several years. Yet, general and lesion specific complications are to be expected during the early postoperative period. The knowledge and understanding of these complications and their physiology is crucial for an optimal management of these patients. Optimal management of the postoperative pediatric cardiac surgical patient requires a thorough understanding of patient anatomy, physiology, surgical repair or palliation, and clinical condition. Postoperative care focuses on anticipating potentially deleterious events and instituting a proactive approach in managing patients. Intervention strategies are directed at preventing low cardiac output and avoiding adverse sequelae in major organ systems. Acute low cardiac output, diastolic disfunction or pulmonary hypertension are some of the most important general complications, whereas residual shunts, residual obstruction of ventricular outflow tracts, or valve disfunction should be expected after some specific heart diseases and surgical procedures. A residual lesion is a lesion that is present at the time of the completion of an operation or intervention. Residual lesions may be secondary to three etiologies: 1) Attempted therapy to treat the lesion may have failed; 2) The lesion may have been intentionally not treated and purposefully left present, and 3) Knowledge of the lesion may not have existed until completion of the operation or intervention.

Postoperative management of pediatric patients undergoing cardiac surgery requires an understanding of basic principles of tissue oxygenation, cardiovascular physiology and anatomy and pathophysiology of congenital 
heart disease. Signs and symptoms of low cardiac output syndrome should be treated timely and aggressively and the diagnoses and treatment strategies should take into account both the general problems of any post-operative cardiac patient as well as the specific heart disease and type of surgery performed.

\section{References}

[1] Krishnamurthy, G., Ratner, V. and Bacha, E. (2013) Neonatal Cardiac Care, a Perspective. Seminars in Thoracic and Cardiovascular Surgery: Pediatric Cardiac Surgery Annual, 16, 21-31. http://dx.doi.org/10.1053/j.pcsu.2013.01.007

[2] Beke, D.M., Braudis, N.J. and Lincoln, P. (2005)Management of the Pediatric Postoperative Cardiac Surgery Patient. Critical Care Nursing Clinics of North America, 17, 405-416. http://dx.doi.org/10.1016/j.ccell.2005.08.006

[3] Miletic, K.G., Spiering, T.J., Delius, R.E., et al. (2014) Use of a Novel Vasoactive-Ventilation-Renal Score to Predict Outcomes after Paediatric Cardiac Surgery. Interactive CardioVascular and Thoracic Surgery, 20, 289-295.

[4] Bronicki, R.A. and Chang, A.C. (2011) Management of the Postoperative Pediatric Cardiac Surgical Patient. Critical Care Medicine, 39, 1974-1984. http://dx.doi.org/10.1097/CCM.0b013e31821b82a6

[5] Pouard, P. and Bojan, M. (2013) Neonatal Cardiopulmonary Bypass. Seminars in Thoracic and Cardiovascular Surgery: Pediatric Cardiac Surgery Annual, 15, 59-61. http://dx.doi.org/10.1053/j.pcsu.2013.01.010

[6] Ungerleider, R.M. (2005) Optimizing Response of the Neonate and Infant to Cardiopulmonary Bypass. Cardiology in the Young, 15, 142-148. http://dx.doi.org/10.1017/S1047951105001186

[7] Wessel, D.L. (1992) Postoperative Management of the Open Heart Surgery Patient. In: Gioia, F.R., Stidham, G.L. and Yeh, T.S., Eds., Pediatric Clinical Care Medicine, 135-152.

[8] Cyran, S.E., Hannon, D.W., Daniels, S.R., Gelfand, M.J., Bailey, W.W., Wilson, J.M. and Kaplan, S. (1987) Predictors of Postoperative Ventricular Dysfunction in Infants Who Have Undergone Primary Repair of a Ventricular Septal Defect. American Heart Journal, 113, 1144-1148. http://dx.doi.org/10.1016/0002-8703(87)90926-4

[9] Kavey, R.E. (2006) Optimal Management Strategies for Patients with Complex Congenital Heart Disease. Circulation, 113, 2569-2571. http://dx.doi.org/10.1161/CIRCULATIONAHA.106.629154

[10] Cullen, S., Shore, D. and Redington, A. (1995) Characterization of Right Ventricular Diastolic Performance after Complete Repair of Tretralogy of Fallot: Restrictive Physiology Predicts Slow Postoperative Recovery. Circulation, 91, 1782-1789. http://dx.doi.org/10.1161/01.CIR.91.6.1782

[11] Jonas, R. (2004) Left Ventricular Outflow Tract Obstruction: Aortic Valve Stenosis, Suaortic Stenosis, Supravalvar Aortic Stenosis. In: Jonas, R., Nardo, D. and James, A., et al., Eds., Comprehensive Surgical Management of Congenital Heart Disease, Atnold, London, 320-340.

[12] Uva, M.S., Galetti, L., Gayet, F.L., Piot, D., Serraf, A., Bruniaux, J., Comas, J., Roussin, R., Touchot, A., Binet, J.P., et al. (1995) Surgery for Congenital Mitral Valve Disease in the First Year of Life. The Journal of Thoracic and Cardiovascular Surgery, 109, 164-174. http://dx.doi.org/10.1016/S0022-5223(95)70432-9

[13] Yoshimura, N., Yamaguchi, M., Oshima, Y., Oka, S., Ootaki, Y., Murakami, H., Tei, T. and Ogawa, K. (1999) Surgery for Mitral Valve Disease in the Pediatric Age Group. The Journal of Thoracic and Cardiovascular Surgery, 118, 99106. http://dx.doi.org/10.1016/S0022-5223(99)70148-0

[14] Vargas, F.J., Mengo, G., Granja, M.A., Gentile, J.A., Rannzini, M.E. and Vazquez, J.C. (1998) Tricuspid Annuloplasty and Ventricular Placation for Ebstein's Malformation. The Annals of Thoracic Surgery, 65, 1755-1757. http://dx.doi.org/10.1016/S0003-4975(98)00290-2

[15] Lipshultz, S.E. (2000) Ventricular Dysfunction Clinical Research in Infants, Children and Adolescents. Progress in Pediatric Cardiology, 12, 1-28. http://dx.doi.org/10.1016/S1058-9813(00)00076-X

[16] Tede, N. and Child, J. (2000) Diastolic Dysfunction in Patients with Congenital Heart Disease. Cardiology Clinics, 18, 491-499. http://dx.doi.org/10.1016/S0733-8651(05)70157-0

[17] Yano, M., Kohno, M., Ohkusa, T., Mochizuki, M., Yamada, J., Kohno, M., et al. (2000) Effect of Milrinone on Left Ventricular Relaxation and Calcium Uptake Function of Cardiac Sarcoplasmic Reticulum. American Journal of Physiology-Heart and Circulatory Physiology, 279, H1898-H1905.

[18] Wessel, D.L., Adatia, I., Giglia, T.M., Thompson, J.E. and Kulik, T.J. (1993) Used of Inhaled Nitric Oxide and Acetylcholine in the Evaluation of Pulmonary Hypertension and Endothelial Function after Cardiopulmonary By-Pass. Circulation, 88, 2128-2138. http://dx.doi.org/10.1161/01.CIR.88.5.2128

[19] Petros, A.J. and Pierce, C.M. (2006) The Management of Pulmonary Hypertension. Pediatric Anesthesia, 16, 816-821.

[20] Balaguru, D., Artman, M. and Auslender, M. (2000) Management of Heart Failure in Children. Current Problems in Pediatrics, 30, 1-35. http://dx.doi.org/10.1016/S0045-9380(00)80035-3

[21] Kay, J.D., Colan, S.D. and Graham Jr., T.P. (2001) Congestive Heart Failure in Pediatric Patients. American Heart 
Journal, 142, 923-928. http://dx.doi.org/10.1067/mhj.2001.119423

[22] Egan, J.R., Clarke, A.J., Williams, S., Cole, A.D., Ayer, J., Jaceobe, S., et al. (2006) Levosimendan for Low Cardiac Output: A Pediatric Experience. Journal of Intensive Care Medicine, 21, 183-187. http://dx.doi.org/10.1177/0885066606287039

[23] Namachivayam, P., Crossland, D.S., Butt, W.W. and Shekerdemian, L.S. (2006) Early Experience with Levosimendan in Children with Ventricular Dysfunction. Pediatric Critical Care Medicine, 7, 445-448. http://dx.doi.org/10.1097/01.PCC.0000235251.14491.75

[24] Camino, M., Riaño, B., Martínez, C. and Zunzunegui, J.L. (2008) Levosimendan Experience in Paediatric Population. Med Clin Monogr (Barc), 9, 18-21. 